\title{
Observaciones de teorías éticas en los procesos argumentativos de trabajadores sociales
}

\section{Ethical theories observations of argumentative processes led by social workers}

\section{Patricio Miranda ${ }^{1}$}

\begin{abstract}
Resumen
El foco de esta investigación lo constituye el rol de las teorias éticas en los procesos decisionales de trabajadores sociales. Se comparan los hallazgos en Chile con los de la investigación de R. Osmo, y R. Landau, realizada en la Paul Baerwald School of Social Work de The Hebrew University of Jerusalem en Israel. Se examinan los argumentos de 103 trabajadores sociales en Chile relativos a sus opciones en términos de principios éticos en general y dilemas éticos en particular.
\end{abstract}

Palabras claves: argumentación ética - valores- decisiones - Trabajo Social

\begin{abstract}
The focus of this investigation is constituted by the role of the ethical theories in the decision making processes of social workers. The results of a research in Chile are compared with the results of the investigation of R. Osmo, and R. Landau, carried out in the Paul Baerwald School Social of Work of The Hebrew University of Jerusalem in Israel. The arguments of 103 social workers in Chile are examined considering their options in terms of general ethical principles and particular ethical dilemmas.
\end{abstract}

Key words: ethical argumentation - values - decisions - Social Work

\section{Introducción}

El foco de esta investigación lo constituye el rol de las teorías éticas en los procesos decisionales de trabajadores sociales. Este vínculo ha sido objeto de una investigación específica en la Paul Baerwald School of Social Work de The Hebrew University of Jerusalem en Israel, desarrollada por R. Osmo, PhD y R. Landau, PhD. Estas autoras centraron su atención en las argumentaciones de trabajadores sociales, observando la correspondencia de sus estructuras argumentativas con formas de teorías éticas específicas, específicamente, de carácter deontológico o teleológico. Los interrogantes directrices de la indagación empírica apuntaban a determinar cómo justifican los trabajadores sociales sus decisiones y elecciones; cómo formulan sus argumentos; cuáles son los términos que utilizan; que relación de derivación guardan tales términos con uno o más esquemas de teorías éticas.
Como se deriva de los interrogantes, se trata de una investigación empírica sobre cuestiones éticas en los procesos argumentativos. Esta delimitación del objeto de investigación la circunscribe en los límites del enfoque empírico- analítico. Razón más que suficiente para cuidar de caer en la clásica falacia naturalista de derivar juicios de valor a partir de juicios de hecho. Desde el momento en que se asume que "lo moral no puede ser identificado con ninguno de los fenómenos naturales que afectan la vida humana" (Cortina \& Martínez, 2001:108), se torna imperativa una vigilancia epistemológica que cuide de la tentación (muy cara a toda forma de empirismo) de resolver cuestiones éticas sustantivas en trabajo social a partir de estudios empíricos de argumentaciones éticas de trabajadores sociales.

Pero la imposibilidad lógica de fundar juicios de valor a partir de juicios de hecho no torna trivial ni irrelevante el análisis empírico de los procesos

1 Subdirector de Docencia. Escuela de Trabajo Social pmirandr@uc.cl 
de argumentación ético. Concordando con Osmo $\&$ Landau en que la percatación de sus propios puntos de vista moral por parte de los trabajadores sociales aparece como un indicio de su mayor o menor reflexividad en su quehacer profesional, indagaciones de esta naturaleza contribuyen a los procesos de elucidación del trabajo social: pensar lo que se hace y saber lo que se piensa (Castoriadis, en Heler,2002).

Para los trabajadores sociales no sólo la conciencia ética es una parte necesaria de su práctica profesional (IFSW, 2004), sino que se espera de ellos que estén preparados para explicar sus decisiones basadas en consideraciones éticas, y que sean responsables de sus elecciones y acciones (IFSW, 2004). Allí precisamente se sitúa el foco de esta indagación, en el escrutinio de las consideraciones éticas que los trabajadores sociales despliegan cuando se ven enfrentados a la necesidad de justificar sus puntos de vista y sus decisiones.

Como anotan Osmo \& Landau, los trabajadores sociales necesitan "identificar sus propios valores personales" (Loewenberg, Dolgoff \& Harrington, 2000, p.133), para comprenderse como seres éticos (Abramson, 1996), para comprometerse en una argumentación explícita que puede hacer una contribución significativa a la calidad de la toma de decisiones (Osmo y Landau, 2001), y para tomar una posición más crítica hacia su trabajo profesional (Gambrill, 1997; Mattison, 2000). Una intervención social reflexiva demanda "el escrutinio explícito de los temas y la justificación explícita de los procesos de toma de decisiones" (Osmo \& Landau, 2004) en cuestiones, problemas y/o dilemas (Banks, 1997). Goldstein "exige que los trabajadores sociales ofrezcan argumentos articulados para sus preferencias en términos de principios éticos cuando se enfrentan con distintos dilemas éticos" (Osmo \& Landau, 2004). Esta explicitación adquiere mayor relieve a la hora de asumir el imperativo ético que para los profesionales significa el tener que dar cuenta de sus intervenciones profesionales, de sus fundamentos, procesos y resultados.

El elucidar sus argumentos en la toma de decisiones expone a los trabajadores sociales al pensamiento crítico y al escrutinio público, así como permite el examen de las deliberaciones implícitas que guían a los profesionales (Osmo \& Landau, 2004) y que aun atemáticamente están teóricamente configuradas. De allí que tenga sentido el solicitar a los profesionales "que justifiquen sus juicios morales" (Banks, 1997:24).
Para el estudio de la argumentación Osmo \& Landau siguen de cerca los desarrollo de Toulmin, para quien "la argumentación es un acto de formación de una racionalidad, de diseño de conclusiones y de su aplicación en discusiones y decisiones" (Toulmin en Osmo \& Landau, 2004). En la perspectiva teórica de Toulmin un argumento se estructura sobre la base de dos componentes principales o postulados: uno que muestre una visión particular y por lo menos uno que especifique razones para esa visión. Un argumento comunica una visión fundada. No es una mera enunciación. "Cuando discutimos, no sólo decimos lo que pensamos, sino también por qué lo pensamos" (Osmo \& Landau, 2004). Un argumento ofrece al interlocutor la posibilidad, o más bien lo pone en situación, de tener que elaborar un juicio crítico, tomar una posición de acuerdo o desacuerdo. La toma de decisiones frente a cuestiones, problemas y/o dilemas éticos (Banks, 1997) se desarrolla sobre la base de procesos de argumentación -si bien no siempre explicitados ni sometidos a la crítica racional. La argumentación es así, "un elemento importante en la toma de decisiones éticas" (Osmo \& Landau, 2004).

El preguntarse por el tipo de perspectivas, pautas o, más precisamente, por el punto de vista moral que los ayudan a evaluar y justificar decisiones concernientes a distintas cuestiones éticos-morales que convergen en su quehacer profesional, constituye -al decir de Rhodes (1998)- un deber para los trabajadores sociales. Cooper (1993) observa que la justificación se refiere al sistema de creencias o teoría que está siendo usado para dar sentido a nuestra forma de vida (Osmo \& Landau, 2004). Pero no ha de entenderse que con el relevar en esta indagación la importancia del estudio de la argumentación se busque con ello reeditar la creencia según la cual "la única ética posible sería la indagación de la lógica de los argumentos morales y el análisis del lenguaje moral" (Cortina, 2001:107).Con esta investigación no se quiere hacer un guiño al empirismo al modo de un Tughendat en el marco de la ética analítica (Sádaba, 1989).

No obstante la importancia que la argumentación tiene para el proceso de toma decisiones éticas, la investigación empírica hecha desde el trabajo social (ver Jansson and Dodd, 1998) sobre argumentación en la toma de decisiones éticas (ver Osmo y Landau, 2001) es aún incipiente. En el caso chileno en particular no se cuenta con un acumulo de investigaciones específicas sobre estos procesos.

Aún cuando en la literatura especializada en temas de ética en trabajo social se pueden reconocer diver- 
sos modelos de toma de decisiones éticas (Reamer, 1990; Rhodes, 1986; Loewenberg, Dolgoff y Harrington, 2000; Congreso, 1999; Matisson, 2000; Sánchez Vidal,1991), aún se sabe muy poco acerca del proceso actual de las argumentaciones de los trabajadores sociales cuando son demandados a justificar sus principios éticos y/o confrontados por dilemas éticos en la práctica (Osmo \& Landau, 2004). ¿Cómo justifican los trabajadores sociales sus decisiones y elecciones? ¿Cómo formulan sus argumentos? ¿Cuáles son los términos que utilizan? ¿Son estos términos consistentes con uno o más marcos de teorías éticas? ¿Difieren estos argumentos según los contextos culturales? Son todos interrogantes para los cuales no se cuenta con estudios especializados.

El esfuerzo de observar teorías éticas en las estructuras argumentativas de trabajadores sociales conecta con la discusión sobre las clasificaciones éticas. De la confrontación entre las teorías éticas en el largo decurso de la razón práctica ha surgido un cúmulo de clasificaciones éticas: descriptivas y normativas, de móviles y de fines, de bienes y de fines, materiales y formales, autónomas y heterónomas, de bienes y de valores, deontológicas y teleológicas, de la intención y de la responsabilidad, cognitivistas y no cognitivistas, naturalistas y no naturalistas, comunitaristas y universalistas, nomológicas y dialógicas, de máximos y de mínimos. Esta diversidad abierta de clasificaciones éticas es reflejo tanto de los diversos enfoques filosóficos como de los diversos aspectos destacados en el fenómeno de la moralidad. Cada una de estas clasificaciones vienen siendo modos lógicos bajos los cuales se busca reconstruir la acción moral (Cortina, 2001:105).

De acuerdo a Osmo \& Landau, las teorías éticas en la literatura del trabajo social pueden ser divididas en dos grupos mayores de manera de ayudar a los trabajadores sociales a reconocer y entender los principios en los cuales están basadas sus decisiones éticas (e.g., Matisson, 2000; Reamer, 2001): (1) Teorías deontológicas, que plantean que ciertos tipos de acción son inherentemente correctas o incorrectas, como una cuestión de principio. Este grupo de teorías se focaliza en satisfacer los propios deberes; respetar los derechos y la autonomía de otros y el tratar a otros con justicia igualitaria. (2) Teorías teleológicas, que postulan que ciertas acciones deben ser realizadas porque son buenas a razón de sus consecuencias. Una orientación teleológica que ha influido al trabajo social es la teoría utilitarista (Reamer, 2001).

Banks (2001) identifica dos tipos de teorías éticas: 81) aquellas que se focalizan en principios de acción, como las orientaciones deontológicas (e.g. respeto por la persona individual y el reconocimiento de los derechos de libertad de los usuarios en la toma de sus propias decisiones y elecciones) y utilitaristas (e.g. utilidad y justicia). (2) aquellas que se focalizan en el carácter de los agentes morales y las relaciones que se dan entre ellos, como la ética de la virtud y la ética del cuidado. Más adelante, Hinman (1994) introduce la teoría ética de los derechos como una de las teorías éticas contemporáneas. Con todo, las teorías éticas que esta investigación supone no agotan el arco semántico de la ética en sus desarrollos actuales. Además ellas no han ser tratadas como recíprocamente excluyentes. ¿Cómo desconocer la dependencia que el desarrollo de la teoría de los derechos ha tenido respecto de la ética kantiana?; por nombrar sólo un caso de solapamiento.

Osmo \& Landau asumieron en su investigación la perspectiva de Banks lo que les permitió ampliar el registro teórico dando cabida a "un número de teorías éticas relevantes para el trabajo social, en adición a aquellas teorías éticas más citadas en la literatura del trabajo social: teorías éticas de la virtud, el cuidado y los derechos" (Osmo \& Landau, 2004).

Cada una de estas teorías, al menos en su versión más fuerte, reclama ser exclusivamente correcta. De acuerdo a Banks la teoría ética kantiana "tiende a enfatizar a la persona individual y sus derechos y deberes, particularmente los principios de libertad y justicia" (Banks, 1997:47). Al decir de Hinman la teoría deontológica alega que "la moralidad es un asunto de tener la intención correcta, una que puede ser deseada universalmente para todos los seres humanos". En tanto, la teoría ética utilitarista -al decir de Banks- "remarca la noción del bien público, prestando atención a las consecuencias de las acciones en relación a los principios de utilidad y justicia" (Banks, 1997:47). Para Hinman, la teoría utilitarista reclama que "la moralidad es solamente un asunto de consecuencias"; la "teoría de los derechos ve los temas morales como temas de derechos y deberes correspondientes para respetar los derechos de otros", y la "teoría de la virtud mantiene que la moralidad es primeramente un asunto de carácter" (Hinman, esto es, el tener las virtudes morales de alguien que actúa por motivos adecuados como el actuar con respeto, y benéficamente y con equidad (Beauchamps \& Childress, 1994). La ética del cuidado, relacionada a la ética de la virtud en algunos aspectos, se focaliza en un conjunto de rasgos que son valorados en relaciones personales cercanas: compasión, simpatía, fidelidad, y así otros (Beauchamps \& Childress, 1994). 
Esta investigación examina los argumentos de trabajadores sociales en Chile para sus preferencias de principios éticos en general y específicamente en el contexto de distintas situaciones que contienen dilemas éticos (profesional y personal). Los argumentos de los trabajadores sociales son examinados en términos de las 5 teorías éticas referidas diferenciando al interior de la deontológica las de carácter monológico y dialógico. Se busca responder las siguientes preguntas:

¿Los argumentos de trabajadores sociales reflejan una o más teorías éticas en sus elecciones de principios éticos?

¿Los argumentos de trabajadores sociales reflejan diferentes teorías éticas en diferentes situaciones prácticas?

¿El contenido de los argumentos de trabajadores sociales es congruente con el contenido de los principios éticos en términos de teorías éticas?

¿Difiere el modo en que se reflejan las teorías éticas en las argumentaciones de trabajadores sociales de Chile e Israel?

La investigación que se llevó a cabo el año 2005, sobre la base de una muestra de 103 trabajadores sociales seleccionados en la Región Metropolitana desde diversos ámbitos de desempeño profesional, requirió a los participantes el ranguear una lista de 12 principios éticos del Trabajo Social, solicitándoles las argumentaciones que justificaban la selección de los tres primeros así como la del último. La compilación de principios, se elaboró a partir de la revisión de bibliografía relevante del trabajo social (Bloom, 1990; Loewenberg, Dolgoff \& Harrington, 2000; Reamer, 2001). Seguidamente, a los trabajadores sociales que componían la muestra, se les presentaron dos dilemas éticos asociados a situaciones prácticas (por ejemplo, embarazo de una adolescente que no desea que su familia conozca su condición); ¿cómo decide aquí el trabajador social? Ante los dilemas, se les requirió a priorizar los principios para su decisión demandándoles simultáneamente las argumentaciones desde las cuales fundamentaban sus decisiones éticas.

\section{Metodología}

\section{Diseño}

En lo fundamental se sigue la metodología que sustentó la investigación de Osmo \& Landau. De este modo se buscó garantizar la comparabilidad en los hallazgos de investigación.
El diseño fue de carácter no experimental, correspondiendo específicamente a una investigación social mediante encuesta (Cea D'Ancona, 2001: 239-291). Los trabajadores sociales seleccionados en la muestra fueron entrevistados en sus trabajos, sobre la base de una adaptación del cuestionario estructurado desarrollado por Osmo \& Landau (2003).

\section{Instrumento}

De manera de asegurar la comparabilidad de los resultados, el instrumento estuvo constituido por un cuestionario semiestructurado basado en el elaborado por Osmo $\&$ Landau. El instrumento adaptado fue validado por el equipo investigador del Israel, no obstante se hizo la observación sobre la posibilidad de que las preguntas iniciales relativas al marco de referencia, pudieran generar algún tipo de sesgo en las argumentaciones.

El instrumento fue sometido a un proceso de pretest del que se derivó la necesidad de algunas modificaciones formales.

La pauta de entrevista elaborada sigue la siguiente estructura:

1. De manera de poder contar con un marco interpretativo de los datos generados en la encuesta, se optó por iniciar la entrevista explorando el marco de referencia de los entrevistados a través de 5 preguntas abiertas relativas a los significados de la ética, y de los principios y dilemas éticos, además de indagar en aquellos que fueran más comunes en el quehacer del trabajador social. Estos resultados serán comunicados en otro artículo.

2. En un segundo ítem de la entrevista, se solicitó a los participantes ranguear una lista de principios adaptada de aquella elaborada por Osmo \& Landau a partir de bibliografía relevante del Trabajo Social (Bloom, 1990; Loewenberg, Dolgoff \& Harrington, 2000; Reamer, 2001). Se les presentaron los doce que se indican:

1. El principio de equidad e inequidad postula que los trabajadores sociales tratan de igual manera a las personas que se hayan en las mismas circunstancias o en situaciones equivalentes; al mismo tiempo, tratan de manera diferente a personas que se hayan en situaciones disímiles, si la inequidad es relevante de ser considerada. 
2. El principio de justicia básica postula que los trabajadores sociales articulan las múltiples necesidades e intereses de los usuarios con las posibilidades y recursos disponibles, conforme a criterios de justicia.

3. El principio de privacidad y confidencialidad postula que los trabajadores sociales tienen en cuenta los principios de derecho a la intimidad, confidencialidad y uso responsable de la información.

4. El principio de protección de la vida postula que los trabajadores sociales reconocen el derecho a la vida como el más básico de todos los derechos.

5. El principio del bien o interés de los individuos postula que los trabajadores sociales trabajan en estrecha colaboración con los clientes y usuarios, y en interés de los mismos, pero prestando el debido respeto a los intereses de las demás personas involucradas.

6. El principio de veracidad y acceso a la información postula que los trabajadores sociales toman decisiones prácticas que les permiten hablar con la verdad y revelar toda información relevante para sus clientes y para otros profesionales.

7. El principio de autonomía y libertad personal postula que los trabajadores sociales respetan y fomentan el derecho de los clientes a la autodeterminación y asisten a los clientes en sus esfuerzos para identificar y clarificar sus objetivos.

8. El principio de calidad de vida postula que los trabajadores sociales promueven una mejor calidad de vida para todas las personas, tanto para los individuos como para la comunidad.

9. El principio de provisión de necesidades humanas básicas postula que los trabajadores sociales orientan su acción a la satisfacción de necesidades humanas básicas.

10. El principio del bien o interés de lo público postula que los trabajadores sociales orientan su acción hacia la provisión de bienes públicos.
11. El principio de menor daño postula que al enfrentarse a dilemas que tienen el potencial de causar algún daño, los trabajadores sociales procuran evitar, prevenir, reducir o reparar dicho daño.

12. El principio de obligación de obedecer la ley y las regulaciones postula que el trabajador social enmarca su quehacer en el respeto a las leyes y regulaciones institucionales.

Los entrevistados debieron indicar el grado de ayuda que cada uno de los principios le prestaba en su quehacer profesional. Asimismo, se les solicitó ordenar en importancia estos doce principios éticos y explicitar las razones del orden escogido para los tres principios que consideraron más importantes y para aquel que consideraron menos importante.

3. Posteriormente, se buscó observar cómo los trabajadores sociales argumentan respecto a dilemas éticos concretos, primero desde un punto de vista profesional y luego desde un punto de vista personal. Aquí el entrevistado se enfrentó a dos situaciones prácticas donde se les requirió priorizar los principios éticos para su decisión demandándoles simultáneamente las argumentaciones desde las cuales fundamentaban su elección de los dos primeros principios escogidos que podían guiarle en la solución del dilema.

El primer dilema ético describía una situación práctica donde una adolescente embarazada de 14 años no quiere que sus padres se enteren de su condición (una versión modificada de un caso descrito en Loewnberg $\&$ Dolgoff, 1992: 40). El segundo dilema describía una familia de tres generaciones donde la abuela de 70 años vive con la familia de su hija(o). Ella se vuelve el centro del conflicto entre la pareja. El único hogar de ancianos que el trabajador social encuentra adecuado es mucho menos deseable para ella que la casa familiar (Loewnberg \& Dolgoff, 1992: 192).

Estas viñetas fueron seleccionadas por Osmo $\&$ Landau porque los dilemas parecían en su contenido adecuados para ser discutidos desde distintas perspectivas éticas. A diferencia de la investigación de estas autoras, ambos dilemas fueron presentados a la totalidad de los entrevistados, debiendo el primero ser abordado desde un punto de vista profesional y personal, y el segundo sólo desde un punto de vista profesional. 
4. Las entrevistas fueron administradas por el investigador asistente y alumnos integrantes del equipo investigador, previamente sometidos a un proceso de entrenamiento.

5. Las entrevistas fueron grabadas y aplicadas entre los meses de agosto y octubre de 2005. La administración de la forma final del cuestionario requirió alrededor de 30 a 40 minutos.

6. La confiabilidad del instrumento y de la construcción de las codificaciones fue determinada sobre la base de categorizaciones cruzadas entre tres miembros del equipo investigador.

\section{Muestra}

El diseño muestral sigue en lo fundamental el de Osmo \& Landau, no obstante se amplió la base empírica. El diseño corresponde a un muestreo estratégico (Cea D'Ancona, 2001:220-221), de carácter no probabilístico. Las unidades muestrales fueron seleccionadas de acuerdo al criterio correspondiente a una muestra constituida por sujetos-tipo (Hernández, 1994: 232), ello atendida su relevancia en investigaciones donde el objetivo es la riqueza, profundidad y calidad de la información y no la cantidad, y estandarización (Hernández, 1994: 232). Para favorecer la comparabilidad con la población objeto de la investigación realizada en Israel, se seleccionó una muestra de tamaño similar (aproximadamente 60) de entre trabajadores sociales que en la realidad chilena se ven enfrentados a dilemas como los descritos. De acuerdo a la realidad de los ámbitos de desempeño profesional de los trabajadores sociales en Chile, se seleccionaron, además, 15 casos de cada uno de los siguientes ámbitos: municipio, empresa, organismos del Estado (ministerios, programas, etc.). La muestra estuvo constituida por trabajadores de la Región Metropolitana. El tamaño muestral final fue de 103 casos.

La distribución por sexo de la muestra es la siguiente: Un $85.6 \%$ es de mujeres y un $14.4 \%$ es de hombres. Este resultado se corresponde con la distribución de la matrícula total de los trabajadores sociales en Chile donde un $85 \%$ son mujeres y un $15 \%$ son hombres (ver en www.futurolaboral.cl).

El $57.8 \%$ de las mujeres declaró estar casadas, $31.3 \%$ solteras, $1.2 \%$ viuda y $9.6 \%$ separada de hecho o anulada.

En el caso de los hombres, un 73,3\% declaró estar casado, un $26.7 \%$ soltero y para las otras categorías no se presentaron casos.
El tiempo promedio que llevaban los informantes en sus trabajos actuales fue de 5.89 años $(\mathrm{SD}=$ 6.425; rango 1 - 27).

Los ámbitos de desempeño laboral se distribuyen de la siguiente manera: $12.4 \%$ en el área de Salud, 10.5\% Red SENAME, 15.2\% Familia, 4.8\% Adultos mayores, 3.8\% municipios, $17.1 \%$ Empresas, 9.5\% Políticas públicas, $6.7 \%$ Área Comunitaria, 5.7\% Área Académica. Un $10.5 \%$ se desempeña en otros ámbitos y de un 3.8\% de la muestra no se obtuvo información.

\section{Análisis de contenido de los argumentos}

Las siguientes instrucciones de codificación fueron especialmente construidas por Osmo \& Landau para analizar los argumentos:

1. Indicar si el informante incluyó razones para el rankeo de un principio ético dado en su argumento.

2. Indicar si el contenido de los argumentos para el rankeo de un principio ético dado se relaciona con una o más de las 5 teorías éticas identificadas: utilitarista; deontológica; de los derechos; de la virtud; del cuidado, o con valores personales o profesionales.

Siguiendo en la línea de Osmo \& Landau, cada declaración en la respuesta fue codificada en forma separada, permitiendo a cada informante aplicar más de un argumento y, consecuentemente, descansar en más de una teoría ética.

Los criterios de codificación que construyeron Osmo $\&$ Landau y el propio equipo investigativo, son los que se indican:

1. El argumento fue codificado como utilitarista si él o parte de él incluía ideas o palabras relacionadas con la utilidad y el consecuencialismo, como la maximización del bien y la minimización del daño, o palabras como ganancia, pérdida y resultados.

2. El argumento fue codificado como deontológico si él o parte de él incluía ideas o palabras relacionadas con el deber, la intención o el imperativo categórico.

3. El argumento fue codificado como basado en derechos si él o parte de él incluía ideas o palabras relacionadas con derechos, elegibilidades, y sus respectivas obligaciones.

4. El argumento fue codificado como basado en virtudes si él o parte de él estaba enfocado en el trabajador social o incluía ideas o palabras relacionadas con su carácter. 
5. El argumento fue codificado dentro de una ética del cuidado si él o parte de él estaba enfocado en el cliente o incluía ideas o palabras relacionadas con la relación profesional y el proceso de cuidado.

6. El argumento fue codificado como basado en valores profesionales cuando la argumentación aludía a elementos identitarios del ethos profesional.

7. El argumento fue codificado como basado en valores personales cuando la argumentación aludía a los propios marcos axiológicos.

La confiabilidad de las instrucciones de codificación fue determinada por el $90 \%$ de consenso entre tres jueces: el investigador principal y dos integrantes del equipo. En una primera etapa se seleccionó una muestra aleatoria de las transcripciones, aplicándoles las instrucciones de codificación y examinando las concordancias y discordancias entre los resultados obtenidos por los jueces. Luego, se seleccionó una segunda muestra aleatoria, repitiendo el procedimiento anterior. Luego, el investigador responsable categorizó las partes de la entrevista correspondientes a los argumentos ofrecidos frente a los principios y dilemas éticos.

Una vez transcritas las entrevistas, se realizó un análisis de contenido de los argumentos, para lo cual se utilizó el software de análisis cualitativo Atlas-ti. Ello permitió generar reportes que contenían las citas seleccionadas en función de las categorías. Sobre esa base se realizaron los últimos afinamientos a la categorización.

Para el objetivo de carácter comparativo entre Chile e Israel 4, se utilizó una matriz cualitativa de comparación.

\section{Resultados}

La mayoría de los informantes entregaron argumentos para sus tres primeros rankings de principios éticos. Un total de 488 argumentos fueron analizados.

Los argumentos relativos a los principios éticos justificados en los cuatro contextos, fueron sometidos a un análisis de contenido. Ellos fueron analizados sin diferenciar entre el primer, el segundo, tercero y cuarto principio preferido -cuando existía-, en tanto los informantes fueron requeridos sólo de dar argumentos para la importancia de los principios que ellos rankearon en los primeros lugares sin especificar la diferencia entre ellos.
Pregunta de investigación 1 - ¿Los argumentos de trabajadores sociales reflejan una o más teorias éticas en sus elecciones de principios éticos?

\section{Distribución según modalidad de argumentación}

Al examinar los argumentos según si se observa una relación de correspondencia con teorías éticas o no, se obtuvieron los siguientes resultados:

TABLA 1

\begin{tabular}{|l|c|}
\hline \multicolumn{2}{|c|}{$\begin{array}{c}\text { Frecuencia según base teórica/no teórica de } \\
\text { argumentación sobre principios }\end{array}$} \\
\hline Base de argumentación & Frecuencia Relativa \\
\hline Teorías éticas & $32.6 \%$ \\
\hline No teórica-ética & $67.4 \%$ \\
\hline Total & $\begin{array}{l}\mathbf{1 0 0} \% \\
(488)\end{array}$ \\
\hline
\end{tabular}

La base de argumentación dominante observada en los discursos de los trabajadores sociales que constituyeron la muestra es 'no teórica'. Al justificar la elección de los principios sólo en un 32\% de los argumentos se observa una relación de adecuación con algunas de las teorías éticas estudiadas.

\section{Distribución según modo de argumentación 'no teórico-ético'}

Al examinar los argumentos en que no se observó una base teórica relativa a algunas de las teorías éticas que consideró esta investigación, se obtuvieron los siguientes resultados:

TABLA 2

\begin{tabular}{|l|c|}
\hline \multicolumn{2}{|c|}{$\begin{array}{c}\text { Frecuencia según base no teórica de } \\
\text { argumentación de principios }\end{array}$} \\
\hline $\begin{array}{l}\text { Base de argumentación } \\
\text { no teórica-ética }\end{array}$ & Frecuencia Relativa \\
\hline Valores personales & $28.9 \%$ \\
\hline Valores profesionales & $52.0 \%$ \\
\hline Otros & $19.1 \%$ \\
\hline Total & $\mathbf{1 0 0 \%}$ \\
& $(329)$ \\
\hline
\end{tabular}

La base dominante de argumentación correspondió a 'valores profesionales' (52\%), seguida de valores personales (28.9\%). Un $19.1 \%$ no ofrece algún tipo de argumentación, las más de las veces sólo hace enunciaciones o descripciones.

\section{Distribución según teorías éticas}

$\mathrm{Al}$ analizar los argumentos que guardan una relación de adecuación con algunas de las teorías éticas estudiadas, se obtuvieron los siguientes resultados: 


\section{TABLA 3}

\begin{tabular}{|l|c|}
\hline \multicolumn{2}{|c|}{ Frecuencias según teorías éticas de base } \\
\hline \multicolumn{1}{|c|}{ Teorías éticas } & Frecuencia \\
\hline Utilitarista & $8.2 \%$ \\
\hline Deontológica & $37.7 \%$ \\
\hline De los derechos & $35.2 \%$ \\
\hline De la virtud & $6.3 \%$ \\
\hline Del cuidado & 12.6 \\
\hline Total & $\begin{array}{l}\mathbf{1 0 0} \% \\
(\mathbf{1 5 9})\end{array}$ \\
\hline
\end{tabular}

Del total de argumentos asociados a teorías éticas la teoría de mayor frecuencia es la deontológica $(37.7 .6 \%)$, seguida por la teoría de los derechos (35.2\%). Con una marcada diferencia de estas teorías se ubican la del cuidado (12.6\%), la utilitarista (8.2\%) y de la virtud (6.3\%).

Pregunta de investigación 2 - ¿Los argumentos de trabajadores sociales reflejan diferentes teorías éticas en diferentes contextos de argumentación?

\section{TABLA 4}

\begin{tabular}{|c|c|c|c|c|c|}
\hline \multicolumn{6}{|c|}{$\begin{array}{l}\text { Frecuencia de los argumentos en } \\
\text { de las teorías éticas en } 4 \text { diferentes co } \\
\text { Caso de Chile. }\end{array}$} \\
\hline \multicolumn{6}{|c|}{ Contextos } \\
\hline Teorías éticas & $A^{*}$ & $\mathrm{~B}^{*}$ & $C^{*}$ & $\mathrm{D}^{*}$ & Total \\
\hline Utilitarista & $\begin{array}{c}11.17 \\
\text { (13) }\end{array}$ & 9.35 & 36.48 & 0.0 & $\begin{array}{c}18.43 \\
(87)\end{array}$ \\
\hline Deontológica & $\begin{array}{c}38.54 \\
(60)\end{array}$ & 30.21 & 37.16 & 33.33 & $\begin{array}{l}35.59 \\
(168)\end{array}$ \\
\hline De derechos & $\begin{array}{c}32.96 \\
(56)\end{array}$ & 16.54 & 6.08 & 16.66 & $\begin{array}{c}18.22 \\
(86)\end{array}$ \\
\hline De la virtud & $\begin{array}{l}8.37 \\
(10)\end{array}$ & 4.31 & 0.67 & 16.66 & $\begin{array}{l}4.87 \\
(23)\end{array}$ \\
\hline Del cuidado & $\begin{array}{l}8.93 \\
(20)\end{array}$ & 39.56 & 19.59 & 33.33 & $\begin{array}{l}21.61 \\
(102)\end{array}$ \\
\hline Total & $\begin{array}{l}100 \% \\
(179)\end{array}$ & $\begin{array}{l}100 \% \\
(139)(\end{array}$ & $\begin{array}{c}100 \% \\
148)\end{array}$ & $\begin{array}{c}100 \% \\
(6)\end{array}$ & $\begin{array}{c}100 \% \\
(472 *)\end{array}$ \\
\hline
\end{tabular}

La tabla 1 muestra que la teoría ética deontológica es la teoría dominante en la justificación de los principios éticos preferidos en el contexto del ranking general $(A=38.54 \%)$, frecuencia que tiende a mantenerse en los argumentos que justifican los principios relacionados a situaciones específicas ( $\mathrm{B}=30.21 \%, C=37.26 \%, \mathrm{D}=33.33 \%$ ).
La teoría utilitarista y la teoría del cuidado aparecen con mayor frecuencia para justificar principios éticos en situaciones específicas. En el caso de la teoría utilitarista, ésta representa un $36.48 \%$ de los argumentos esgrimidos en el caso de la familia de tres generaciones, y sólo un $11.17 \%$ de aquellos presentados en el contexto del ranking general. Este porcentaje es similar al 8.93\% de argumentos que corresponden a la teoría del cuidado en el contexto del ranking general, teoría que sin embargo aparece con mayor frecuencia -junto a la teoría kantiana para el contexto D- en la justificación de argumentos respecto a la adolescente embarazada, tanto en la dimensión profesional (37.16\%), como personal (33.3\%).

Los argumentos referidos a la teoría de la virtud, están mínimamente representados tanto en el ranking general como en las situaciones específicas ( $A=8.37 \%$, $\mathrm{B}=4.31 \%, \mathrm{C}=0.67 \%$ ); a excepción del contexto $\mathrm{D}$ donde aparece con un $16.66 \%$ de frecuencia.

La teoría de los derechos muestra amplias diferencias entre el ranking general de principios y las situaciones específicas, apareciendo con mayor frecuencia en la justificación general de principios éticos (32.96\%), que en los contextos referidos a la familia de tres generaciones (6.08\%) y al embarazo adolescente ( $\mathrm{B}=16.54 \%)$, en tanto que en $\mathrm{D}$ alcanza un porcentaje de $16.66 \%$.

Al igual que en la investigación realizada en Israel no fue encontrada ninguna diferencia notable en la clasificación de teorías éticas a través de la comparación de los argumentos entre la evaluación profesional y la personal en el caso de la adolescente embarazada (B y D) (Osmo \& Landau, 2004).

En resumen, los resultados muestran que el contenido de los argumentos de los trabajadores sociales, en términos de teorías éticas, cambia según cambia el contexto de las situaciones prácticas, a excepción de la teoría deontológica que aparece con similar frecuencia en la totalidad de los contextos ( $A=38.59 \%$, $\mathrm{B}=30.21 \%, \mathrm{C}=37.16 \%, \mathrm{D}=33.33 \%$ ).

Pregunta de investigación 4 - ¿Difiere el modo en que se reflejan las teorías éticas en las argumentaciones de trabajadores sociales de Chile e lsrael?

Al comparar las argumentaciones que calificaron como de base teórica, se obtuvieron los siguientes resultados:

\footnotetext{
* Los porcentajes han sido calculados sobre la base de un total de 472 argumentos. El ajuste al total de 488 no se espera que modifique las tendencias observadas.

$A=$ Justificación de principios; $B=$ adolescente embarazada (dilema profesional); $C=$ familia de tres generaciones (dilema profesional); $D=$ adolescente embarazada (dilema personal). Número de participantes $=103$.
} 
TABLA 5

\begin{tabular}{|c|c|c|c|c|c|c|c|c|c|c|}
\hline \multicolumn{11}{|c|}{ Teorías éticas según contexto de argumentación y país } \\
\hline \multirow{3}{*}{ Teorías éticas } & \multicolumn{10}{|c|}{ Contextos } \\
\hline & \multicolumn{2}{|c|}{$A^{*}$} & \multicolumn{2}{|c|}{$\mathrm{B}^{*}$} & \multicolumn{2}{|c|}{$C^{*}$} & \multicolumn{2}{|c|}{$\mathrm{D}^{*}$} & \multicolumn{2}{|c|}{ Total } \\
\hline & Israel & Chile & Israel & Chile & Israel & Chile & Israel & Chile & Israel & Chile \\
\hline Utilitarista & 28 & 11.2 & 36 & 9.4 & 48 & 36.5 & 37 & 0.0 & $\begin{array}{c}35 \\
(115)\end{array}$ & $\begin{array}{l}18.4 \\
(87)\end{array}$ \\
\hline Deontológica & 33 & 38.5 & 23 & 30.2 & 22 & 37.2 & 24 & 33.3 & $\begin{array}{c}27 \\
(88)\end{array}$ & $\begin{array}{l}35.6 \\
(168)\end{array}$ \\
\hline De los Derechos & 11 & 33 & 15 & 16.5 & 12 & 6.1 & 10 & 16.7 & $\begin{array}{c}12 \\
(41)\end{array}$ & $\begin{array}{l}19.5 \\
(92)\end{array}$ \\
\hline De la virtud & 21 & 8.4 & 11 & 4.3 & 10 & 0.7 & 10 & 16.7 & $\begin{array}{c}15 \\
(48)\end{array}$ & $\begin{array}{l}4.9 \\
(23)\end{array}$ \\
\hline Del cuidado & 7 & 8.9 & 15 & 39.6 & 8 & 19.6 & 19 & 33.3 & $\begin{array}{c}11 \\
(37)\end{array}$ & $\begin{array}{c}21.6 \\
(102)\end{array}$ \\
\hline Total & $\begin{array}{l}100 \% \\
(123)\end{array}$ & $\begin{array}{l}100 \% \\
(179)\end{array}$ & $\begin{array}{l}100 \% \\
(114)\end{array}$ & $\begin{array}{l}100 \% \\
(139)\end{array}$ & $\begin{array}{c}100 \% \\
(50)\end{array}$ & $\begin{array}{l}100 \% \\
(148)\end{array}$ & $\begin{array}{c}100 \% \\
(42)\end{array}$ & $\begin{array}{c}100 \% \\
(6)\end{array}$ & $\begin{array}{l}100 \% \\
(329)\end{array}$ & $\begin{array}{l}100 \% \\
\left(472^{*}\right)\end{array}$ \\
\hline
\end{tabular}

*Los porcentajes han sido calculados sobre la base de un total de 472 argumentos. El ajuste al total de 488 no se espera que modifique las tendencias observadas.

Respecto a la totalidad de los argumentos, la Tabla 5 muestra que la teoría de base más frecuente para el caso de Israel es el utilitarismo (35\%), mientras en el caso de Chile la mayor frecuencia de los argumentos es congruente con la teoría deontológica (35.6\%). Los porcentajes que siguen a estas mayorías corresponden a distintas teorías según el país. En el caso de Israel la segunda mayoría corresponde a la teoría deontológica (27\%), mientras en el caso de Chile ésta corresponde a la teoría del cuidado (21.6\%).

Respecto a esta última el porcentaje de argumentos en el caso de Israel es sólo de un 11\%, distancia que también se hace visible respecto a la teoría utilitarista en el caso de Chile, donde sólo un 18.4\% del total de argumentos es congruente con la teoría utilitarista.

Diferencia similar se presenta en el caso de la teoría de la virtud, donde mientras en Israel el porcentaje corresponde a un $15 \%$, en el caso de Chile éste llega a un $4.9 \%$.

La diferencia disminuye respecto a la teoría de los derechos, donde los argumentos de Israel relacionados con esta teoría corresponden a un $12 \%$ y los de Chile a un $18.2 \%$.

En el contexto del ranking general de principios, en ambos países la teoría deontológica sobredetermina con mayor frecuencia la justificación de las argumentaciones sobre la elección de principios
(Israel: 33\%; Chile: 38.5\%). Sin embargo, los países difieren en la teoría que sucede a la deontológica, correspondiendo al utilitarismo en el caso de Israel (28\%), y a la teoría de los derechos en el caso de Chile (33\%). En este contexto, en Chile el utilitarismo aparece con un 11.2\%, mientras en Israel la teoría de los derechos lo hace con un porcentaje similar (11\%).

En ambos países, las teorías que son priorizadas en el contexto del ranking general, no necesariamente son luego escogidas para las situaciones específicas.

Israel y Chile coinciden en basarse con frecuencia en categorías utilitaristas para argumentar en torno al dilema de la familia de tres generaciones (48\% y $36.5 \%$, respectivamente). Difieren en las otras dos situaciones específicas referidas al embarazo adolescente, donde la mayoría en Israel sigue correspondiendo a la teoría utilitarista ( $\mathrm{B}=36 \%$; $\mathrm{D}=37 \%$ ) mientras en Chile la teoría del cuidado pasa ser la primera prioridad ( $B=39.6 ; \mathrm{D}=33.3 \%)$. En el caso del último contexto, la teoría del cuidado comparte la primera preferencia con la teoría deontológica (33.3\%).

En términos de la clasificación por teoría ética, la teoría utilitarista aparece con frecuencia en los 4 contextos para el caso de Israel ( $\mathrm{A}=28 \%, \mathrm{~B}=36 \%$, $\mathrm{C}=48 \%, \mathrm{D}=37 \%$ ), mientras que para Chile esta es sólo priorizada con frecuencia en el contexto de la familia de 3 generaciones (36.5\%). 
Respecto a la teoría deontológica, en los 4 contextos los porcentajes son mayores en el caso de Chile que en el de Israel correspondiendo en promedio a un tercio de los argumentos expuestos.

En el ámbito de la teoría de los derechos, se produce una diferencia notoria entre ambos países, específicamente en el contexto del ranking general. Mientras sólo un 11\% de los argumentos ofrecidos por trabajadores sociales de Israel se relaciona con la teoría de los derechos, este porcentaje llega a un 33\% en el caso de Chile.

Para la teoría de la virtud, en general, es mayor el número de argumentos congruentes con ella en el caso de Israel. Especialmente en el contexto del ranking general, donde Israel alcanza un 21\% y Chile un $8.4 \%$. Del total de argumentos compatibles con la ética de la virtud se observa una diferencia de 10 puntos porcentuales entre trabajadores sociales de Israel y Chile.

Similar a lo ocurrido con la teoría deontológica, en los 4 contextos la teoría del cuidado aparece con mayor frecuencia en el caso de Chile que en el de Israel. Esta diferencia no es tan notoria en el ranking de principios éticos (Israel 7\%, Chile 8.9\%), pero sí en las dos situaciones referidas a la adolescente embarazada (Israel $\mathrm{B}=15 \%, \mathrm{D}=19 \%$; Chile $\mathrm{B}=39.6 \%$, $\mathrm{D}=33.3 \%$ ).

Los resultados muestran que en ambos países el contenido de los argumentos de los trabajadores sociales, en términos de teorías éticas, cambian con el contexto de las situaciones prácticas.

\section{Discusión y conclusión}

Este estudio se focalizó en la comparación de los argumentos provistos por trabajadores sociales de Chile e Israel en términos de su correspondencia con teorías éticas, cuando ellos son confrontados a justificar su selección de principios éticos y su postura ante dilemas éticos profesionales. Junto con la investigación de Osmo y Landau nuestros resultados iluminan un área relativamente desconocida en el proceso de toma de decisiones éticas en la práctica de los trabajadores sociales. Este desconocimiento específico se condice con el estado de la investigación sobre cuestiones éticas en el trabajo social. En efecto, si bien la discusión sobre ética y valores ha tenido lugar desde que la profesión se inicia formalmente en el siglo XIX, la deliberación y el estudio sistemático de los conflictos éticos de los trabajadores sociales es más reciente (Reamer, 1995:898). Es a partir de 1970 que se observa un renovado interés en examinar los valores de los mismos trabajadores sociales y los aspectos éticos de su práctica (Reamer, 1995:895). En el periodo anterior, es posible observar una enorme laguna, un formidable vacío en la producción intelectual en el ámbito de la ética en trabajo social (Netto, 1996:6).

Como se desprende de los resultados a un nivel agregado, en los argumentos generados en los distintos contextos de argumentación se observan diferencias apreciables (no se ha calculado aún la significación estadística) entre Chile e Israel. Allí donde en Israel la teoría dominante es la utilitarista (35\%) en Chile ese lugar lo ocupa la teoría deontológica (la utilitarista alcanza sólo un 18.4\%). Ambas teorías, como anota Nidia Aylwin se reconocen entre las de mayor influjo en el tratamiento de las cuestiones ética en trabajo social (Aylwin, 1993). "Para analizar el contexto ético en la profesión de Trabajo Social, es necesario referirse a las principales corrientes de pensamiento que han predominado en la filosofía contemporánea, y que también están presentes en las profesiones, respecto a la forma de justificar las decisiones éticas. Ellas son las teorías deontológicas y teleológicas, que forman parte de la ética normativa" (Aylwin, 1993:42).

Allí donde las teorías utilitaristas han puesto el foco en las consecuencias de las decisiones y acciones procurando la mayor utilidad (o bienestar en términos habituales en trabajo social), pudiendo llegar a decirse que los principios utilitaristas han sido tradicionalmente la guía más popular para la realización de decisiones éticas (Osmo \& Landau, 2004; Aylwin, 1993), las teorías éticas de corte kantiano han capturado el fenómeno de la moralidad sobre el cimiento de los imperativos categóricos, que permiten responder la pregunta sobre qué se debe (pregunta por lo correcto) y no sobre qué debemos hacer (pregunta por lo bueno) (Habermas, 2000).

La diferencia observada entre Chile e Israel talvez se explique por el tradicional pragmatismo de la tradición anglosajona (humus del utilitarismo) que en Chile parece haber tenido un menor influjo. Como es sabido, una de las tradiciones dominantes en la filosofía moral (ética) es la tradición anglosajona de indole empirista. Bebe esa tradición de autores como Locke, Hume o Mill. Como anota Vial Larraín, "en una línea de clara continuidad con el pensamiento de Hume está la ética dominante en Gran Bretaña en el siglo XIX, el utilitarismo, que representa John Milll y que prolonga en él siglo XX el llamado consecuencialismo" (Vial Larraín,1998:78). A esta tradición se adscriben el utilitarismo, el emotivis- 
mo, el consecuencialismo. Pero, ¿qué explicaría el peso de lo deontológico en Chile? Una posibilidad es que guarde relación con la expansión del ethos de la modernidad (Salvat,2002).

Que la ética del cuidado, en el caso de Chile, ocupe la segunda posición (21.6\%) al nivel general de la argumentación y la primera posición (39.6\%) al nivel de los argumentos asociados al dilema del embarazo adolescente, es una interrogante cuya elucidación desborda el carácter prelimimar de este informe de investigación. Recuérdese, sin embargo, que en el caso de Israel la ética del cuidado ocupa la última posición (11\%) a nivel general y comparte las posiciones $3^{\mathrm{a}}$ y $4^{\mathrm{a}}$ con le ética de los derechos (15\%) en el caso del dilema del embarazo adolescente. ¿Qué podría explicar esta diferencia? ¿Factores culturales? ¿Tradiciones distintas de trabajo social interpenetradas por estructuras y culturales irreductibles entre sí? ¿O será una ironía de la contingencia rortyana? ¿Guardará relación con la situación de permanente conflictividad en que se encuentra Israel? ¿Presionará ese hecho a una mayor sensibilización ante las consecuencias de las acciones y decisiones? No lo sabemos y no estamos en condiciones de adelantar alguna hipótesis más allá de una genérica apelación a las diferencias de los mundos vitales en los que se inserta el trabajo social.

Otra diferencia importante con Israel dice relación con que allí la teoría de los derechos, la teoría de la virtud y la teoría del cuidado fueron observadas con menor frecuencia de la esperada (Osmo \& Landau, 2004: 15). No ocurre lo mismo en el caso de la investigación realizada en Chile. De hecho, la teoría del cuidado es la segunda en frecuencia y la de los derechos ocupa la tercera posición de entre cinco. ¿Cómo explicar en el caso de Chile el peso de la teoría ética de cuidado, segunda a nivel general y primera a nivel del dilema de la embarazada adolescente? ¿Qué dice del trabajo social en Chile? ¿Estará asociada con imaginarios tradicionales de trabajo social signados por un fuerte énfasis asistencialita? o ¿es señal de apertura a ámbitos del fenómeno de la moralidad descuidados en el trabajo profesional? Se recordará que las teorías éticas del cuidado se focalizan en un conjunto de rasgos que son valorados en relaciones personales cercanas: compasión, simpatía, fidelidad, y así otros (Beauchamps \& Childress, 1994). Como anota Habermas, "las éticas de la compasión se han percatado de que esta profunda vulnerabilidad [de las personas] hace necesaria una garantía del recíproco respeto" (Habermas, 2000:19). Talvez por ello, Nouwen ha llamado la atención sobre una comprensión reducida de la compasión. Partiendo de la semántica originaria del concepto, derivado de las palabras latinas pati y cum, que juntas significan "sufrir con", sostendrá que "la compasión significa una total inmersión en la condición humana. Cuando miramos la compasión así, entonces queda claro que ella implica algo más que una genérica amabilidad o ternura del corazón" (Nouwen,1996:16). Reformulando la intuición de la ética de la compasión, Habermas postula, junto al principio de justicia (igual respeto y iguales derechos para cada individuo particular), el principio de solidaridad que exige empatía y preocupación por el bienestar del prójimo" (Habermas, 2001:20). ¿La centralidad de la ética del cuidado en el caso de Chile será expresión de esta empatía y preocupación por la suerte del otro? Y si fuera así, ¿por qué se expresa con menor intensidad en el caso del dilema relativo al adulto mayor? (allí aparece en tercera posición con un 19.6\%).

Tampoco deja de ser significativo que, a diferencia de Israel, nuestros hallazgos exhiban una mayor preponderancia de la teoría de los derechos para el caso de Chile. ¿Refeljo de una conciencia madurada al compás del compromiso histórico de muchos trabajadores sociales en Chile con la defensa de los derechos humanos?

Otro de los hallazgos destacados por Osmo y Landau, es que su investigación provee soportes adicionales a lo que ya Loewnberg, Dolgoff y Harringtons habían sostenido, en el sentido de que los trabajadores sociales son deontológicos a nivel de los principios y consecuencialistas a nivel de la práctica profesional (Osmo \& Landau, 2004: 16). Nuestros hallazgos no son del todo compatibles con dicha tesis. Efectivamente, en el caso de Chile la distribución de las teorías éticas difiere al cambiar de contexto argumentativo. Los trabajadores sociales investigados aparecen como deontológicos en los principios pero difiriendo de orientación teórica al cambiar de contexto argumentativo. Efectivamente, al nivel del quehacer profesional se observa un quiebre de la tendencia al pasar del dilema de la adolescente embarazada, al de la situación familiar generada con la presencia del adulto mayor. En relación con el primer dilema, predomina la ética del cuidado (39.6\%), en tanto que con relación al segundo dilema, la orientación es hacia el utilitarismo (36.5\%). ¿Qué explica esta diferencia más hallá del apresuramiento de pensar en una inconsistencia?

El análisis de contenido de los argumentos, entregado por los participantes en diferentes contextos, 
muestra que la mayor frecuencia de argumentos de los trabajadores sociales entrevistados para este estudio basaron sus argumentos en conceptos deontológico, seguido a 14 puntos porcentuales de diferencia por conceptos relativos a la ética del cuidado (21.6\%) y muy de cerca por la ética de los derechos (19.5\%) y el utilitarismo (18.4\%). Con una frecuencia mucho menor $(4.9 \%)$ se ofrecieron argumentos compatibles con la ética de la virtud. ¿Sugieren estos resultados que la imagen del trabajador social de sí mismo/a pesa poco en general a la hora de tomar decisiones? ¿Habría que trabajar esta dimensión en la formación ética de los futuros trabajadores sociales?

Nuestros resultados no confirman el planteamiento de Loewnberg, Dolgoff \& Harrington (2000) respecto a que los trabajadores sociales son deontológicos en los principios pero adoptan una postura utilitarista en la práctica. Carr (1999) plantea que es natural preguntar dónde y cuándo es apropiado razonar desde un punto de vista deontológico o utilitarista. La única respuesta general a esta pregunta es que debe ser contextualmente determinado. Banks (2001) también sostiene que ni las teorías deontólógicas ni las utilitaristas "pueden proveernos de un principio último para determinar lo correcto o incorrecto de las acciones" (p. 34, mientras Beauchamps y Childress (1994) escriben que hay una "teoría moral común" que es pluralista y combina varias teorías éticas. De hecho, la inclinación común en la ética profesional es ampliar la perspectiva de las teorías éticas desde una postura dicotómica (deontológica y teleológica) hacia un acercamiento más pluralista y diferencial, es decir, teorías que enfatizan diferentes aspectos de los dilemas éticos (Brannigan y Boss, 2001). A diferencia de Israel, nuestros resultados, muestran que la teoría de los derechos, la teoría de la virtud y la teoría del cuidado fueron utilizadas en mayor frecuencia que lo esperado. El mayor porcentaje asociado a conceptos de la teoría de los derechos apareció en el contexto de la adolescente embarazada como un dilema profesional (16.54\%), en conflicto con el derecho de la adolescente de manejar su vida como un derecho básico que el profesional debe respetar. Los conceptos de la teoría de la virtud formaron un $8.4 \%$ en la jerarquía general de los principios éticos y aquellos de la teoría del cuidado un $39.6 \%$ en la jerarquía de principios éticos relativo a la adolescente embarazada desde un punto de vista personal.

Aún cuando los participantes en este estudio no fueron demandados de justificar sus preferencias por los principios éticos en términos de teorías éticas, nuestro análisis de contenido de sus argumentos mostrarán si los conceptos de teorías éticas eran identificables y congruentes con los principios éticos preferidos.

Mientras ninguna teoría ética ofrece la verdad completa a un dilema moral, una diversidad de teorías éticas parece dar una herramienta más comprensiva para un análisis efectivo de problemas y dilemas éticos. Concordando implícitamente con Cortina (2001) en cuanto a que, en cuestiones de ética aplicada, las teorías éticas no ofrecen una completa guía para la acción, Osmo \& Landau postulan un pluralismo ético sosteniendo que diversas teorías éticas pueden dar una herramienta más comprensiva para el análisis de dilemas éticos (Osmo \& Landau, 2004: 17-18). De alguna manera, todas las teorías éticas pueden aportar al iluminar distintos determinantes de la acción, a proveer bases racionales para mejor hacer decisiones morales (Osmo \& Landau, 2004: 18). Por su parte, Boss (1998) sugiere adoptar una postura muldimensional que emerge de las fortalezas de cada teoría: "Todas las teorías tienen el mismo objetivo final de proveer una base racional para tomar mejores decisiones morales" (p. 40). En tanto las éticas tienen un rol capital en la comprensión y justificación de la acción moral, una de sus tareas ineludibles "es la de dar razón de fenómeno moral, esto es fundamentarlo (Cortina,2991:130) Tratándose de la ética aplicada -sostendrá Cortina-, "un solo modelo de ética es impotente para orientar las decisiones de los mundos políticos y económico, médico, ecológico o, simplemente, la convivencia ciudadana" (2001:159). Debido a que las teorías éticas pueden ayudar a la toma de decisiones éticas, concordamos con Osmo $\&$ Landau en avalar las recomendaciones de la literatura del trabajo social que plantea que las teorías éticas debieran ser enseñadas en los procesos formativos de los trabajadores sociales (Osmo \& Landau, 2004; e.g. Rhodes, 1998; Matisson, 2000).

También concordamos con Osmo \& Landau en sugerir que las teorías éticas sean estudiadas críticamente. Esto no sólo prepara a los estudiantes para aplicar teorías éticas mientras consideran posibles intervenciones, sino que también les enseña las limitaciones de cada teoría en la explicación y resolución de dilemas éticos. Por ejemplo, como los argumentos de la mayoría de los informantes relacionados con la teoría ética utilitarista, los estudiantes debieran saber que no es fácil decidir el valor de una acción cuando no está claro cuánta 
utilidad es suficiente y cómo la utilidad es medida en términos de consecuencias, dados los límites de predicción (Hinman, 1994; cit. en Osmo \& Landau, 2004). Tampoco la utilidad necesariamente considera la dignidad y los derechos de cada individuo (Brannigan \& Boss, 2001, p. 27. De hecho, el utilitarismo clásico, cuando es llevado al extremo, puede justificar el atropello sobre los derechos de una minoría vulnerable con el fin de beneficiar a la mayoría (Reamer, 2001, p. 28). La aplicación de tales teorías éticas a las decisiones profesionales de la vida real, puede significar una valiosa guía, y de esta manera impulsar una práctica más reflexiva.

Y que el utilitarismo ha de ser estudiado críticamente es algo que no pasó inadvertido a Nidia Aylwin. Para ella, el utilitarismo en la práctica entra con frecuencia en contradicción con los derechos humanos. "El utilitarismo tiene gran influencia en la actualidad porque calza muy bien con la economía de mercado y su búsqueda de la eficiencia. Sin embargo, uno de los problemas más serios que presenta este enfoque ético tiene que ver con la justicia, pues justifica la subordinación de los derechos de una minoría para el logro de un total mayor de bienes. "La idea de justicia, la idea de que alguien posee un derecho que, a diferencia de los derechos de propiedad, no es transable en el mercado, parece introducir una perspectiva no consecuencialista" (Gómez - Lobo, p.22) en el juicio moral de los utilitaristas" (Aylwin, 1993).

Los estudiantes, y particularmente los practicantes, deben ser capaces de discutir críticamente el razonamiento tras sus decisiones discrecionales y ser capaces de sostener el escrutinio y el examen de colegas profesionales e instancias legales, justificando sus decisiones discrecionales en términos de estándares y valores profesionales. Esto es, sus justificaciones éticas deben estar amarradas a justificaciones normativas profesionales y estar en congruencia con lo que puede ser llamado el trabajador social "razonable". La necesidad de desarrollar la autoconciencia y la habilidad de articular explícitamente sus pensamientos, puede probar la importancia de la eliminación de instancias de decisiones arbitrarias y discrecionales que pueden desatender los valores y preferencias de los clientes (Osmo \& Landau, 2004).

A diferencia de Israel, un 91.3\% de los trabajadores sociales del estudio dieron argumentos para sus principios éticos preferidos (en el caso de Israel este porcentaje alcanza el 70\%), permitiéndonos asumir que ellos se muestran competentes en justificar sus decisiones éticas. Sin embargo, el caso es que sólo en el 32.6\% de los argumentos se observa una relación de adecuación con alguna (s) de las teorías éticas consideradas en el estudio. Lo que resulta consistente con la precariedad reflexiva sobre las cuestiones éticas en trabajo social (Reamer,1995; Neto,1996). Esta dificultad puede emerger debido a que los trabajadores sociales no están acostumbrados a someterse a un proceso explicito de justificación de sus acciones y decisiones en situaciones reales.

Situándose las éticas profesionales en el ámbito de las éticas aplicadas, el recurso a la "reflexión y al lenguaje filosófico" (Cortina, 2003a: 21), resulta imprescindible. Precisamente porque la ética en Trabajo Social cuenta con la reflexión filosófica, "no es moral cotidiana, sino aplicación a los problemas cotidianos de lo ganado en los procesos éticos de fundamentación. Por eso, el utillaje que utiliza para comprender y debatir las cuestiones es el propio del kantismo, utilitarismo o el aristotelismo" (Cortina, 2003a: 21). Consiguientemente, importa que el profesional que trabaja las cuestiones éticas en Trabajo Social, "conozca a fondo las distintas teorías éticas (clásicas y contemporáneas) y tenga el discernimiento suficiente como para dilucidar en cada caso cual de ellas resulta más fecunda para ayudar en la toma de decisiones" (Cortina, 2003: 23).

El tratamiento de las cuestiones éticas en trabajo social en el presente exige una confrontación con los nuevos desarrollos en el debate ético internacional cruzado por tensiones entre universalismo y contextualismo, entre liberalismo y comunitarismo, entre otras (Cortina,2001). Las propuestas contemporáneas en trabajo social (Matus,1999) también presionan hacia una relaboración de los discursos que busque hacerse cargo de la pluralidad de voces teóricas desde las cuales se piensa y gestiona la intervención social en sociedades signadas por procesos de complejización y exclusión social. La articulación de estos dos vectores da origen a distintas matrices disciplinares en las que se entreveran concepciones de ética y concepciones de trabajo social. El modo en que se piense la ética sobredetermina el modo en que se piense y configure el trabajo social. Sólo a título de ilustración baste con recordar que para Silva Barroco pensar la ética en trabajo social en el horizonte de una ética tradicional, condujo, en el caso de Brasil, a una moralización de la cuestión social (2001:79). ¿Qué articulaciones pueden pensarse en un presente que se ha vuelto complejo e incierto?

El discernimiento ético de las nuevas intervenciones sociales en este escenario de transformaciones 
se torna él mismo un imperativo ético ineludible. Las profundas mutaciones socio-culturales obligan a repensar tanto las formas de convivencia como los principios éticos que la orientan. La larga historia de compromiso con la justicia social y con el trato justo a los sujetos vulnerados, exige al trabajo social una ampliación de perspectivas. Si "el trabajo social trata con problemas individuales y sociales y con situaciones que son, por definición, difíciles y complejas" (Osmo \& Landau, 2004), hoy esta complejidad ha adquirido ribetes inesperados e inciertos. Para tomar decisiones éticamente fundadas los trabajadores sociales requieren desarrollar procesos de 'elucidación'. El pensar lo que hacen y saber lo que piensan, pasa por el despliegue de argumentaciones fundadas, no sólo al nivel del discurso moral, sino sobre todo al nivel del discurso ético. Estudiar las estructuras argumentativas que comunican los trabajadores sociales enfrentados a cuestiones éticas en su quehacer profesional en correspondencia con teorías éticas contemporáneas se constituye así en un espacio privilegiado de indagación.

Tanto las decantaciones históricas del ethos profesional como los marcos axiológicos y normativos de los mismos trabajadores sociales se ven tensionados con la conciencia de la contingencia del propio punto de vista moral. Es más, aún cuando los valores profesionales de los trabajadores sociales pueden ser en parte compatibles con los valores sociales, pueden haber importantes diferencias en énfasis, prioridades o interpretaciones (Loewenberg, Dolgoff \& Harrington, en Osmo \& Landau, 2004). Es así que en una sociedad pluralista, axiológicamente politeísta en términos weberianos, los trabadores sociales deben hacer un esfuerzo consciente para evaluar continuamente sus valores profesionales y reconstruirlos en las estructuras de su existencia concreta diaria (Cooper, en Osmo \& Landau, 2004). De ahí que, en cada situación, los trabajadores sociales deban decidir nuevamente no sólo cuál principio ético profesional tiene prioridad con respecto a quién (Hugman and Smith, en Osmo \& Landau, 2004), sino desde qué teorías éticas configura sus propias intervenciones.

Como sujetos individualizados en la trama densa de mundos de la vida, "la perspectiva con la cual los trabajadores sociales ven el mundo refleja su filosofía personal y sistema de valores que derivan, a su vez, de su historia personal y cultural" (ver Abramson, en Osmo \& Landau, 2004). La percatación de sus propios puntos de vista moral aparece como un indicio de la mayor o menor reflexividad de los trabajadores sociales en su quehacer profesional.

Por últimos, el reconocerse en un punto de vista moral posibilita la articulación crítica entre los valores profesionales y los valores personales. Los resultados de Osmo y Landau indican que, mientras los trabajadores sociales parecen atribuir gran importancia a los principios éticos profesionales, sus valores personales pueden jugar un gran rol en su proceso de toma de decisiones éticas. La influencia de los valores personales de los trabajadores sociales en su proceso de toma de decisiones requiere aún de un mayor análisis (Osmo \& Landau, 2004).

\section{Bibliografía}

ALLEN, JO ANN. (1995). The Constructivist Paradigm: Values and Ethics. Encyclopedia of Social Work, Washington: National Association of Social Workers.

AYLWIN, N. (1993) Apuntes ética en trabajo social. Santiago de Chile: Archivo Escuela de Trabajo Social Pontificia Universidad Católica de Chile.

AYLWIN, NIDIA. (1996). El Olvido de la Persona. Revista de Trabajo Social, 67.

BANKS, SARAH. (1997). Ética y valores en el trabajo social. Barcelona: Paidós.

BONETTI, DILSÉA et al (ed). (1996). Serviço Social e Etica: Convite a uma Nova Praxis, Sao Paulo: Cortez Editora.

BRANT DE CARVALHO FALCAO, MARÍA DO CARMO, (1997). Serviço Social. Una Visao Teórica, Sao Paulo: Cortez\&Moraez.

CEA D'ANCONA, MA ÁNGELES. (2001). Metodología cuantitativa. Estrategias y técnicas de investigación social. Madrid: Editorial Síntesis.

CORTINA, A. Ética sin moral. Madrid: Tecnos.

CORTINA, A. \& García-Marzá D. (2003). Razón pública y éticas aplicadas. Los caminos de la razón práctica en una sociedad pluralista. Madrid: Tecnos.

FEDERACIÓN INTERNACIONAL DE TRABAJADORES SOCIALES (IFSW). (2004). Ethics in Social Work, Statement of Principles.

FLECK-ANDERSON, ANN. (1998). The Family as Moral Community: A Social Work Perspective. Families in Society, 79 (3) : 233-240.

GOLDSTEIN, HOWARD. (1998). Education for Ethical Dilemmas in Social Work Practice. Families in Society, 79 (3) : 241-253. 
HABERMAS, JURGEN. (2000). Aclaraciones a la ética del discurso. Salamanca: Trotta.

IAMAMOTO, MARILDA V. (1996). O debate contemporáneo do Serviço Social e a Ética Profissional. En BONETTI, Dilséa et al., Serviço Social e Ética. Sao Paulo: Cortez Editora.

LINZER, NORMAN. (1998). Resolving Ethical Dilemmas in Social Work Practice. Boston: Allyn and Bacon.

LOEWENBERG, F.M. (2005). Ethical decisions for social work practice, $7^{\text {a }}$ Ed. Illinois: Broooks/Cole.

MANNING, SUSAN. (1997). The Social Worker as Moral Citizen: Ethics in Action. Social Work, 42 (3).

MATUS, TERESA. (1999). Propuesta contemporáneas en trabajo social. Buenos Aires: Espacio.

NATIONAL ASSOCIATION OF SOCIAL WORKERS. (1995). Encyclopedia of Social Work. 19th ed. Washington: NASW.

NOUWEN, HENRI. (1996). La compasión en la vida cotidiana. Buenos Aires: Lumen.

NETTO, JOSÉ PAULO. (1996). Ética e Crisis dos Projetos de Transformaçao Social. Sao Paulo: Cortez Editora.
OSMO, RUJLA \& LANDAU, RUTH. (2004). The role of ethical theories in decision making by social workers. Israel : [s.n]

REAMER, FREDERIC. (1995). Ethics And Values. En Encyclopedia of Social Work, Washington: NASW.

REAMER, F. G. (1990) Ethical dilemmas in social service, $2 \mathrm{~d}$ ed., Columbia: University Press, New York.

HELER, MARIO. (2002). Filosofía Social \& Trabajo Social. Buenos Aires: Biblos.

SÁNCHEZ VIDAL. ALIPIO. (1999). Ética de la intervención social. Buenos Aires: Paidós.

SILVA BARROCO, MARIA LUCIA. (1996). Bases Filosóficas para una Reflexao sobre Ética e Serviço Social. En: BONETTI, et al., Seviço e Ética. Sao Paulo: Cortez Editora.

TOULMIN, S., RIECKE, \& JANIK, (1984) An introduction to reasoning, Macmillan, New York.

VIAL CORREA, JUAN DE DIOS. (1998). Filosofía moral. Santiago de Chile: Ediciones Universidad Católica de Chile.

FECHA DE RECEPCIÓN: noviembre 2005

FECHA DE ACEPTACIÓN: marzo 2006 